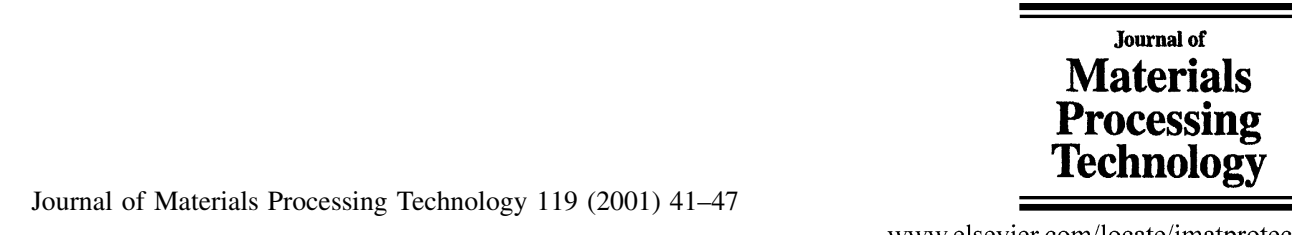

www.elsevier.com/locate/jmatprotec

\title{
Advances in FE explicit formulation for simulation of metalforming processes
}

\author{
J. Rojek ${ }^{\mathrm{a},{ }^{*}}$, O.C. Zienkiewicz ${ }^{\mathrm{b}}$, E. Oñate ${ }^{\mathrm{c}}$, E. Postek $^{\mathrm{a}}$ \\ ${ }^{\mathrm{a}}$ Institute of Fundamental Technological Research, Polish Academy of Sciences, ul. Swietokryska 21, PL-00049 Warsaw, Poland \\ ${ }^{\mathrm{b}}$ University of Wales, Swansea, UK \\ ${ }^{\mathrm{c}}$ International Center for Numerical Methods in Engineering, Barcelona, Spain
}

\begin{abstract}
This paper presents some advances of finite element explicit formulation for simulation of metal forming processes. Because of their computational efficiency, finite element programs based on the explicit dynamic formulation proved to be a very attractive tool for the simulation of metal forming processes. The use of explicit programs in the sheet forming simulation is quite common, the possibilities of these codes in bulk forming simulation in our opinion are still not exploited sufficiently. In our paper, we will consider aspects of bulk forming simulation.

We will present new formulations and algorithms developed for bulk metal forming within the explicit formulation. An extension of a finite element code for the thermomechanical coupled analysis is discussed. A new thermomechanical constitutive model developed by the authors and implemented in the program is presented.

A new formulation based on the so-called split algorithm allows us to use linear triangular and tetrahedral elements in the analysis of large plastic deformations characteristic to forming processes. Linear triangles and tetrahedra have many advantages over quadrilateral and hexahedral elements. Linear triangles and tetrahedra based on the standard formulations exhibit volumetric locking and are not suitable for large plastic strain simulation. The new formulation allows to overcome this difficulty.

New formulations and algorithms have been implemented in the finite element code Stampack developed at the International Centre for Numerical Methods in Engineering in Barcelona. Numerical examples illustrate some of the possibilities of the finite element code developed and validate new algorithms. (C) 2001 Elsevier Science B.V. All rights reserved.
\end{abstract}

Keywords: Explicit formulation; Forming simulation; Split algorithm

\section{Introduction}

Finite element programs based on the explicit dynamic formulation proved to be a very attractive tool for the simulation of metalforming processes. The explicit time integration schemes deal with the system of discretized equations of motion in the following form:

$\mathbf{M} \ddot{\mathbf{r}}+\mathbf{D} \dot{\mathbf{r}}=\mathbf{f}-\mathbf{p}$

where $\mathbf{M}$ and $\mathbf{D}$ are the mass and damping matrices, $\mathbf{r}$ the nodal displacement vector, and $\mathbf{f}$ and $\mathbf{p}$ the vectors of external and internal nodal forces, respectively. Employing Eq. (1) for the known configuration at time $t_{n}$, the solution for the next time instant $t_{n+1}=t_{n}+\Delta t$ is obtained. The effectiveness of the explicit formulation is based on the

\footnotetext{
* Corresponding author.

E-mail address: jrojek@zmit1.ippt.gov.pl (J. Rojek).
}

use of a diagonal mass matrix. There are also disadvantages, the main one is the limitation of the time step due to the conditional stability. The numerical efficiency, however, and other advantages of explicit programs such as low memory requirements and easy treatment of contact conditions make this approach to dominate over implicit methods in industrial applications.

The computation times, however, in case of large industrial problems are still quite long. The implementation of a new triangular shell element as well as the use of techniques of parallel computations reduced simulation times considerably.

The use of explicit finite element programs in the analysis of sheet metal forming has become quite common, while the possibilities of this approach in the simulation of bulk forming seem not to be exploited sufficiently as yet. These problems require taking into account the thermal effects in the deformation process that can be achieved by the coupled thermomechanical analysis. 


\section{Explicit thermomechanical analysis}

\subsection{Discretized equations of the coupled thermomechanical problem}

Simulation of bulk forming processes usually requires taking into account the thermal effects and interaction of thermal and mechanical phenomena. The algorithm for a coupled thermomechanical analysis has been implemented in our explicit program, making it possible to analyse such forming processes.

In the solution of a thermomechanical problem, the solution of the mechanical problem (1) is coupled with the solution of the heat conduction problem governed by the following discretized equation:

$\mathrm{CT}+\mathrm{KT}=\mathrm{Q}$

where $\mathbf{C}$ is the heat capacity matrix, $\mathbf{K}$ the heat conductivity matrix, $\mathbf{Q}$ the heat flux and sources vector, and $\mathbf{T}$ the vector of nodal temperatures. Eqs. (1) and (2) are solved under adequate boundary conditions. Eq. (2) should be supplemented with thermal boundary conditions including heat convection and radiation, thermal effects on the tool surface should be taken into account as well. The forward Euler

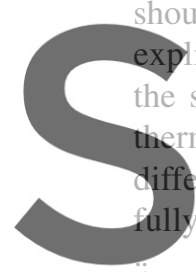

xplicit time integration meth

the solution of Eq. (2) in the

thermomechanical analysis. Th

ifference time integration of Eq

$\dot{\mathbf{r}}_{n}=\mathbf{M}_{\mathrm{D}}^{-1}\left(\mathbf{f}_{n}-\mathbf{p}_{n}-\mathbf{D} \dot{\mathbf{r}}_{n}\right), \quad$ where $\mathbf{M}_{\mathrm{D}}=\operatorname{diag} \mathbf{M}$

(3)

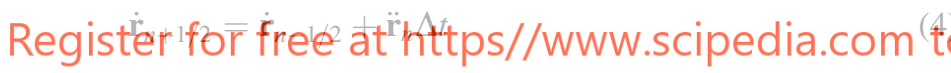 \\ $\mathbf{r}_{n+1}=\mathbf{r}_{n}+\dot{\mathbf{r}}_{n+1 / 2} \Delta t$ \\ $\mathbf{T}_{n+1}=\mathbf{T}_{n}+\mathbf{C}_{\mathrm{D}}^{-\mathrm{i}}\left(\mathbf{Q}_{n}-\mathbf{K} \mathbf{T}_{n}\right) \Delta t, \quad$ where $\mathbf{C}_{\mathrm{D}}=\operatorname{diag} \mathbf{C}$}

The new configuration $\mathbf{r}_{n+1}$ is obtained from the explicit equations of motion with the temperatures assumed fixed, and the new temperature $\mathbf{T}_{n+1}$ is calculated at constant geometry. The results are exchanged at each step and coupling terms are calculated. Eqs. (1) and (2) for the thermomechanical problem are coupled by considering the following effects:

1. Heat generation by the plastic dissipation.

2. Contribution of the thermal expansion to the total material deformation.

3. Influence of the temperature on the yield stress of the material.

The rate of heat generation $q$ due to the plastic dissipation (contributing to the vector $\mathbf{Q}$ ) can be calculated as

$q=\chi \boldsymbol{\sigma}: \mathbf{d}^{\mathrm{p}}$

where $\sigma$ is the Cauchy stress tensor, $\mathbf{d}^{\mathrm{p}}$ the rate of plastic deformation tensor, and $\chi$ the fraction of plastic work converted to heat. The method of accounting for the thermal effects in the constitutive model developed is presented in the next section.

\subsection{Thermo-elastoplastic constitutive model}

A new thermo-elastoplastic model has been developed and implemented in the explicit dynamic code Stampack.
The model employs the concept of hyperelasticity The main
advantage of hyperelastic models compared to hypoelastic
formulations is that therd is no need for calcutation of
derivatives satisfying the criteria of objectivity and no need
for inter ration of the constitutive equations $[2,7]$.

Formulation of the constitutive model for the thermo-

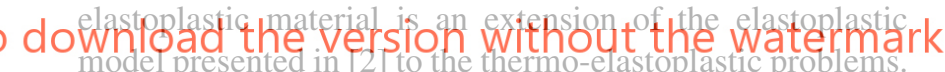

In the description of large thermo-elastoplastic deformations, we assume the multiplicative decomposition (see Fig. 1) of the deformation gradient tensor $\mathbf{F}$ into its elastic, thermal and plastic parts, $\mathbf{F}^{\mathrm{e}}, \mathbf{F}^{\theta}$ and $\mathbf{F}^{\mathrm{p}}$, respectively:

$\mathbf{F}=\mathbf{F}^{\mathrm{e}} \mathbf{F}^{\theta} \mathbf{F}^{\mathrm{p}}$

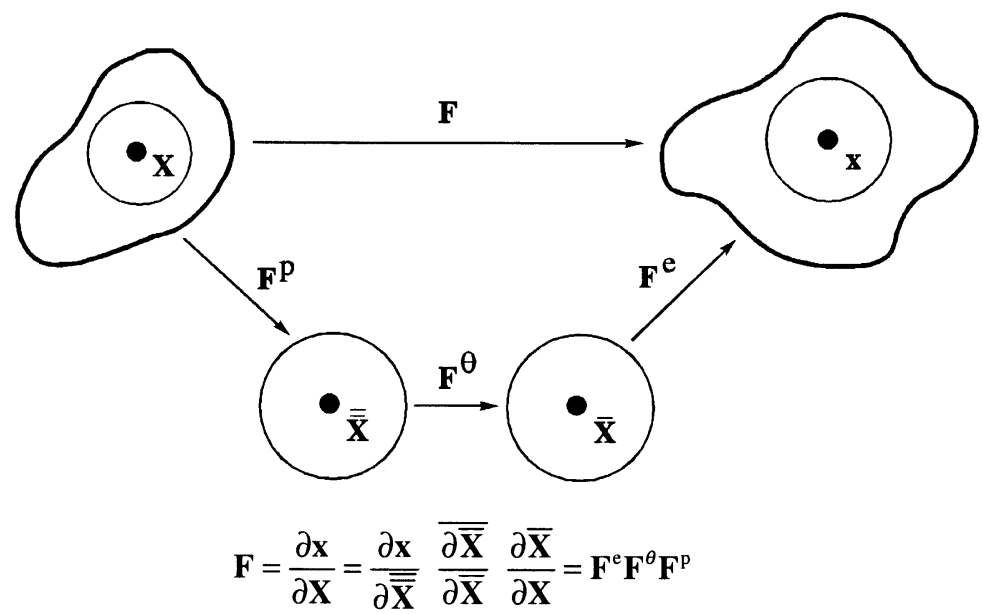

Fig. 1. Multiplicative decomposition of the deformation gradient tensor. 
with

$\operatorname{det} \mathbf{F}^{\mathrm{p}}=1$

$\mathbf{F}^{\theta}=J^{\theta} \mathbf{I}, \quad$ where $J^{\theta}=\mathrm{e}^{3 \alpha\left(T-T_{0}\right)}$

The Almansi strain tensor e can be expressed by means of the deformation gradient tensor $\mathbf{F}$ as

$\mathbf{e}=\frac{1}{2}\left(\mathbf{I}-\mathbf{F}^{-\mathrm{T}} \mathbf{F}^{-1}\right)$

Analogical to Eq. (11), we define the elastic Almansi tensor $\mathbf{e}^{\mathrm{e}}$ (in the spatial configuration) and the thermal Almansi tensor $\overline{\mathbf{e}}^{\theta}$ (in the intermediate configuration):

$\mathbf{e}^{\mathrm{e}}=\frac{1}{2}\left(\mathbf{I}-\mathbf{F}^{\mathrm{e}^{-\mathrm{T}}} \mathbf{F}^{\mathrm{e}^{-1}}\right)$

$\overline{\mathbf{e}}^{\theta}=\frac{1}{2}\left(\mathbf{I}-\mathbf{F}^{\theta^{-\mathrm{T}}} \mathbf{F}^{\theta^{-1}}\right)$

After transforming the thermal Almansi tensor to the spatial configuration (the push-forward operation):

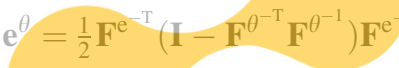

we can introduce the following additive relation:

$\mathrm{e}=\mathrm{e}^{\mathrm{e}}+\mathrm{e}^{\theta}+\mathrm{e}^{\mathrm{p}}$

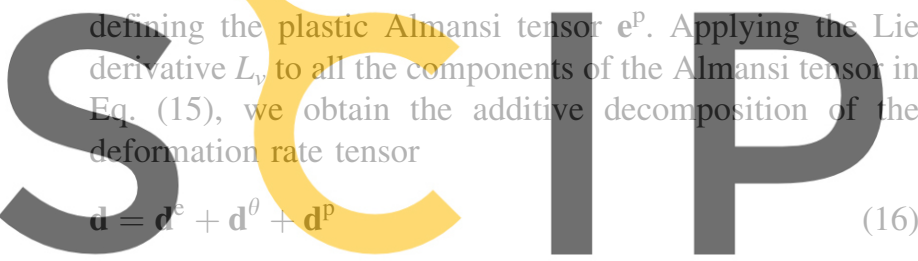

The stress response is characterised by means of the elastic Register for free at htthe form

$\psi^{\mathrm{e}}=\frac{1}{2} \lambda \operatorname{tr}\left(\mathbf{e}^{\mathrm{e}}\right)+\mu\left(\mathbf{e}^{\mathrm{e}}: \mathbf{e}^{\mathrm{e}}\right)$

where $\lambda$ and $\mu$ are the Lamé constants. With this form of the elastic potential the constitutive relation obtained for the Cauchy stresses is following:

$\boldsymbol{\sigma}=\frac{\partial \psi\left(\mathbf{e}^{\mathrm{e}}\right)}{\partial \mathbf{e}^{\mathrm{e}}}=\lambda \operatorname{tr} \mathbf{e}^{\mathrm{e}}+2 \mu \mathbf{e}^{\mathrm{e}}$

The stresses are calculated in a two-step algorithm, the first step is the elastic predictor, and the second one the plastic corrector employing the radial return. For the plastic deformation, the associated flow rule is assumed:

$\mathbf{d}^{\mathrm{p}}=L_{v}\left(\mathbf{e}^{\mathrm{p}}\right)=\dot{\lambda} \frac{\partial f}{\partial \sigma}$

with the Von Mises yield condition:

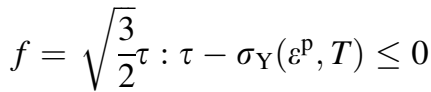

that accounts for the isotropic hardening and thermal softening:

$\sigma_{\mathrm{Y}}=\left[\sigma_{0}+\left(\sigma_{\infty}-\sigma_{0}\right)\left(1-\mathrm{e}^{-\delta_{\varepsilon^{\mathrm{p}}}}\right)+H \varepsilon^{\mathrm{p}}\right]\left[1-H_{\theta}\left(T-T_{0}\right)\right]$ where $\tau$ is the deviatoric Cauchy stress, tensor $\sigma_{0}$ and $\sigma_{\infty}$ the initial and the final yield stresses, $\delta$ the saturation constant, $H$ the hardening modulus and $H_{\theta}$ the thermal softening modulus.

The form of the elastic potential (17) is based on the assumption that the elastic part of the strains $\mathbf{e}_{\mathrm{e}}$ is small, which is fully justified for metals. It is assumed for the reasons of simplicity and efficiency. Some authors starting from the same basic assumption expressed by Eq. (8), cf. [8-10], have developed more general models, considering the possibility of large elastic deformations. Our formulation is simpler in implementation and no loss of accuracy in the considered class of problems is expected. A good behaviour of this model in the problems of metal forming without accounting for thermal effects has been confirmed earlier, cf. [2,3].

\subsection{Finite element implementation}

The developed thermo-elastoplastic model has been implemented in the program Stampack with a 4-node plane strain and axisymmetric element and with an 8-node hexahedral element for three-dimensional analysis. In both the elements, the mixed formulation (with constant pressure)

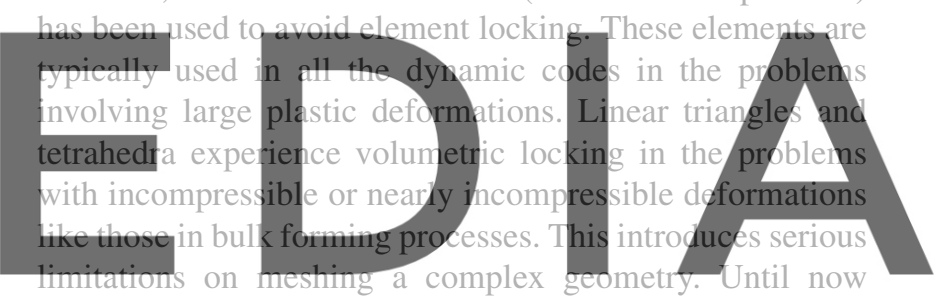

meshing programs work better with triangular and tetrahe-

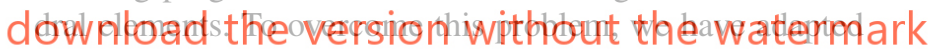
the so-called split allowing us to use linear triangles and tetrahedra [13]. The formulation of the split algorithm is presented below.

\section{Linear triangles and tetrahedra within the split algorithm}

\subsection{Finite element formulations for incompressible problems}

Finite elements based on the standard displacement formulation are vulnerable to volumetric locking in the analysis of problems with incompressible deformations. This poses serious problems in the simulation of elasto-plastic cases. Introduction of a small compressibility does not yield a solution, elements that do not perform well in an incompressible state do not give good results in nearly incompressible state either.

Different methods have been developed to overcome this problem. Some of them employ the mixed finite element formulation in which we split the stresses $\boldsymbol{\sigma}$ into the deviatoric part $\tau$ and pressure $p$ :

$\boldsymbol{\sigma}=\tau+\mathbf{I} p$ 
Then using independent interpolations for displacements $\mathbf{r}=\mathbf{N}_{\mathrm{d}} \overline{\mathbf{r}}$ and pressure $p=\mathbf{N}_{\mathrm{p}} \overline{\mathbf{p}}$ ( $\mathbf{N}_{\mathrm{d}}$ and $\mathbf{N}_{\mathrm{p}}$ are shape functions, $\overline{\mathbf{r}}$ and $\overline{\mathbf{p}}$ the nodal values of displacements and pressure), we obtain the following set of mixed equations cf. $[9,10]$ :

$$
\left[\begin{array}{cc}
\mathbf{K} & \mathbf{Q} \\
\mathbf{Q}^{\mathrm{T}} & \mathbf{G}
\end{array}\right]\left[\begin{array}{l}
\overline{\mathbf{r}} \\
\overline{\mathbf{p}}
\end{array}\right]=\left[\begin{array}{l}
\mathbf{f}_{\mathrm{d}} \\
\mathbf{f}_{\mathrm{p}}
\end{array}\right]
$$

In compressible case $\mathbf{G} \neq \mathbf{0}$ and $\overline{\mathbf{p}}$ can be eliminated:

$$
\left(\mathbf{K}-\mathbf{Q G}^{-1} \mathbf{Q}^{-\mathrm{T}}\right) \overline{\mathbf{r}}=\mathbf{f}_{\mathrm{d}}-\mathbf{Q G}^{-1} \mathbf{f}_{\mathrm{p}}
$$

If pressure is discontinuous between elements, $\overline{\mathbf{p}}$ can be eliminated at the element level.

In incompressible state $\mathbf{G}=\mathbf{0}$, the following equation for pressure can be obtained:

\section{$\left(\mathbf{Q}^{\mathrm{T}} \mathbf{K}^{-1} \mathbf{Q}\right) \overline{\mathrm{p}}=\mathbf{Q}^{\mathrm{T}} \mathbf{K}^{-1} \mathbf{f}_{\mathrm{d}}-\mathbf{f}_{\mathrm{p}}$}

Not all combinations of displacement and pressure interpolations $\left(\mathbf{N}_{\mathrm{d}}\right.$ and $\left.\mathbf{N}_{\mathrm{p}}\right)$ are allowed since some of them render the coefficient matrix $\mathbf{Q}^{\mathrm{T}} \mathbf{K}^{-1} \mathbf{Q}$ singular. Then the effect of volumetric locking appears. This happens in case of linear triangles and tetrahedra with equal order interpolation of displacement and pressure. A mathematical theory has been

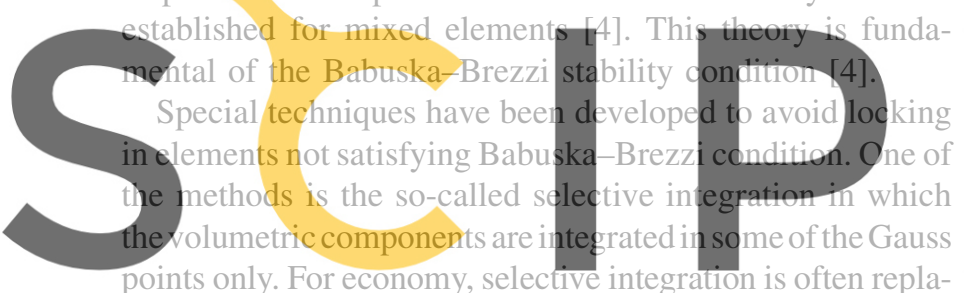
points only. For economy, selective integration is often repla-

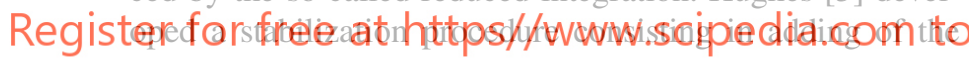
equilibrium equation suitably weighted by the shape functions to the mass conservation equation. Bonet [1] derived for explicit dynamic applications a linear tetrahedron free of volumetric locking using the idea of averaging nodal pressure.

Our stabilising procedure is based on the split algorithms developed in fluid mechanics $[11,12]$. Appropriate splitting of the equations of motion allows equal order interpolation to be used in incompressible flows. Adapting this method to solid mechanics and explicit dynamic program, we have obtained 3-node triangular and 4-node tetrahedral elements that can be used in nearly incompressible problems of bulk metal forming. The basic equations of the split algorithm are presented below, more details has been given by Zienkiewicz et al. [13].

\subsection{The split algorithm}

The split operator will be applied to the Stokes equations for nearly incompressible flow:

$\rho_{0} \frac{\partial v_{i}}{\partial t}=\frac{\partial \tau_{i j}}{\partial x_{j}}-\frac{\partial p}{\partial x_{i}}+g_{i}$

$\frac{1}{c^{2}} \frac{\partial p}{\partial t}=-\rho_{0} \frac{\partial v_{i}}{\partial x_{i}}$ where $\rho_{0}$ is the density, $v_{i}$ the velocity in the $i$ th direction, $\tau_{i j}$ the deviatoric stress component, $p$ the pressure (or mean stress), $g_{\mathrm{i}}$ the $i$ th component of body force, and $c$ the speed of sound given by the relation $c=\sqrt{K} / \rho_{0}, K$ being the bulk modulus. After discretization the Stokes equation can be written in the following form $[9,10]$ :

$$
\left[\begin{array}{cc}
\mathbf{M} & \mathbf{0} \\
\mathbf{0} & \widetilde{\mathbf{M}}
\end{array}\right] \frac{\mathrm{d}}{\mathrm{d} t}\left[\begin{array}{c}
\overline{\mathbf{v}} \\
\overline{\mathbf{p}}
\end{array}\right]+\left[\begin{array}{cc}
\mathbf{K} & \mathbf{Q} \\
\mathbf{Q}^{\mathrm{T}} & \mathbf{0}
\end{array}\right]\left[\begin{array}{c}
\overline{\mathbf{v}} \\
\overline{\mathbf{p}}
\end{array}\right]=\left[\begin{array}{c}
\mathbf{f}_{\mathrm{d}} \\
\mathbf{f}_{\mathrm{p}}
\end{array}\right]
$$

In the above, the standard finite element notation is used $[9,10]$.

A clear analogy between Eqs. (23) and (28) can be noted here. Similarly like in case of Eq. (23) for incompressible problem, the zero diagonal term in the second matrix of Eq. (28) leads to volumetric locking. The splitting algorithm removes this problem.

In the described algorithm, Eq. (28) is split into parts in such a way, however, that the sum of the parts gives the original equation (28). Let us rewrite Eq. (28) in the following form:

$$
\begin{aligned}
& \mathrm{M} \dot{\overline{\mathrm{v}}}=\mathrm{f}_{0}-\mathbf{K} \overline{\mathrm{v}}-\mathbf{Q} \overline{\mathrm{p}} \\
& \widetilde{\mathrm{M}} \bar{p}=-\mathbf{Q}^{\mathrm{T}} \overline{\mathrm{v}}
\end{aligned}
$$

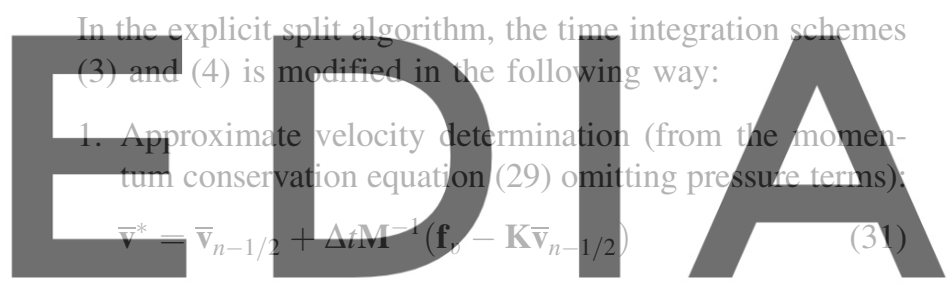

2. The pressure evaluation (from the mass conservation

\section{download the version without the watermark $\overline{\mathbf{p}}_{n+1}=\overline{\mathbf{p}}_{n}-\Delta t \mathbf{M}^{-1}\left(\mathbf{Q} \overline{\mathbf{v}}^{*}-\Delta t \mathbf{H} \overline{\mathbf{p}}_{n}\right)$ \\ where $\mathrm{HI}$ is the standard discretization of the Laplacian operator.}

3 . The velocity correction (from the momentum conservation equation (29) taking into account step 1):

$\overline{\mathbf{v}}_{n+1 / 2}=\overline{\mathbf{v}}^{*}-\Delta t \mathbf{M}^{-1} \mathbf{Q} \overline{\mathbf{p}}_{n}$

The described explicit split algorithm implemented for linear triangles and tetrahedra has been successfully applied to thermomechanical analysis. Test examples of bulk forming are included in the paper.

\subsection{Why is the Babuska-Brezzi condition circumvented?}

When steady state conditions are reached, we have

$\overline{\mathbf{v}}_{n+1 / 2}=\overline{\mathbf{v}}_{n-1 / 2}=\overline{\mathbf{v}}, \quad \overline{\mathbf{p}}_{n+1}=\overline{\mathbf{p}}_{n}=\overline{\mathbf{p}}$

After substituting relations (34) into Eqs. (30)-(32) and eliminating $\overline{\mathbf{v}}^{*}$ we obtain the system of the following form

$$
\left[\begin{array}{cc}
\mathbf{K} & \mathbf{Q} \\
\mathbf{Q}^{\mathrm{T}} & \Delta t(\mathbf{H}-\mathbf{Q M Q})
\end{array}\right]\left[\begin{array}{c}
\overline{\mathbf{v}} \\
\overline{\mathbf{p}}
\end{array}\right]=\left[\begin{array}{c}
\mathbf{f}_{\mathrm{d}} \\
\mathbf{0}
\end{array}\right]
$$

The term on the diagonal involving the variable $\overline{\mathbf{p}}$ is no longer zero. This illustrates the stabilizing effect of the split 
algorithm. The proof that the equation set (35) is well conditioned can be found in [11].

\section{Numerical examples}

\subsection{Impact of a cylindrical bar}

The example studied here is that of an impact with a rigid surface of a cylindrical rod moving with a high speed. This problem is frequently studied by explicit dynamics codes. This example is aimed to show the correctness of the split formulation with linear triangles and tetrahedra that will be used for metal forming simulation.

The material properties for an elasto-plastic model are: modulus of elasticity, $117 \mathrm{GPa}$; Poisson ratio, $v=0.35$, initial uniaxial yield stress, $\sigma_{0}=0.4 \mathrm{GPa}$; and hardening modulus, $H=0.1 \mathrm{GPa}$. The initial length of the bar is $32.4 \mathrm{~mm}$ and the initial radius is $3.2 \mathrm{~mm}$. A solution is obtained for an initial velocity of $227 \mathrm{~m} / \mathrm{s}$. The interval of $80 \mu$ s has been analysed which allows the body to reach a steady state. The final deformed shapes obtained using different formulations are shown in Fig. 2. The results of the

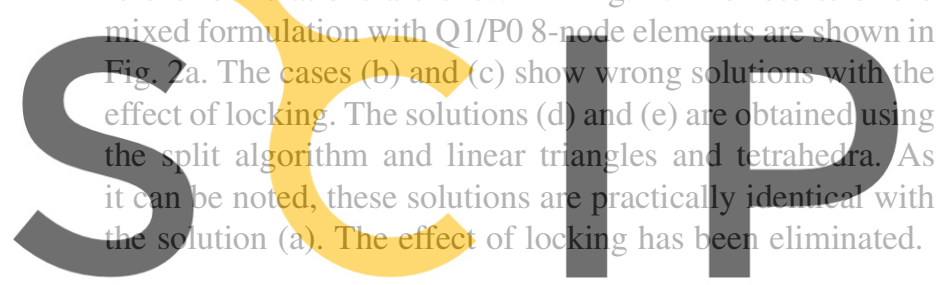

4.2. Upsetting of a cylindrical billet

Register for free at https//www.scipedia.com to d This example was used to verify the new thermoelastoplastic constitutive model and to test the algorithm of thermomechanical analysis implemented in the program Stampack. A cylindrical billet, $30 \mathrm{~mm}$ high and with a radius of $10 \mathrm{~mm}$, is compressed along its axis between two rigid and rough plates. All the surfaces are assumed to be fully insulated. The problem has been defined originally by Lippmann [6] and was analysed in [14]. The analysis was carried out to $60 \%$ upsetting. The mechanical and thermal properties were the following: Young's modulus: $E=2 \times 10^{5} \mathrm{MPa}$, Poisson's coefficient: $v=0.3$, density: $\rho=7830 \mathrm{~kg} / \mathrm{m}^{3}$, yield stress: $\sigma_{0}=\sigma_{\infty}=700 \mathrm{MPa}$, hardening modulus: $H=300 \mathrm{MPa}$, thermal softening modulus: $H_{\theta}=0.002^{\circ} \mathrm{C}^{-1}$, specific heat: $c=586 \mathrm{~J} / \mathrm{kg}{ }^{\circ} \mathrm{C}$, conductivity: $k=52 \mathrm{~W} / \mathrm{m}^{\circ} \mathrm{C}$. The material properties were basically the same as those used in [14] except that thermal softening was considered and rate-independent plasticity was assumed in our models. The final deformation was obtained in $0.1 \mathrm{~s}$.

Both two- and three-dimensional simulation were carried out. In 2D two models were studied, a half of the billet was discretized with 144 quadrilateral and 288 triangular axisymmetric elements, the latter case was analysed using the split algorithm. The results of the analysis for both cases are shown in Fig. 3. Fig. 3 shows the final deformed shapes with the temperature distribution. Similar results have been obtained with quadrilateral and triangular mesh. The results

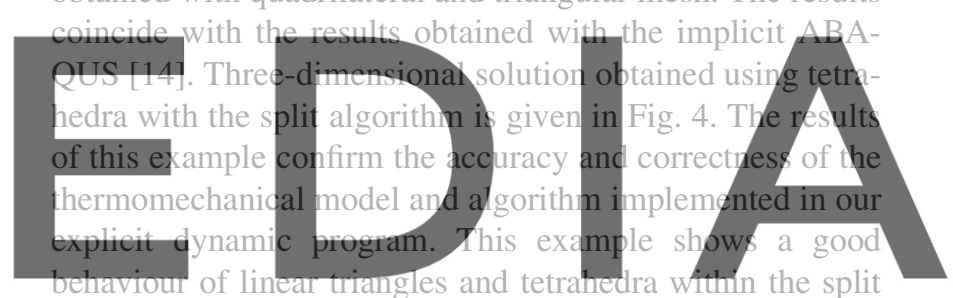

algorithm in the thermomechanical coupled analysis.

\section{download the version without the watermark 4.3. Sidepressing of a cylinder}

A cylinder $100 \mathrm{~mm}$ long with a radius of $100 \mathrm{~mm}$ is subjected to sidepressing between two plane dies. It is

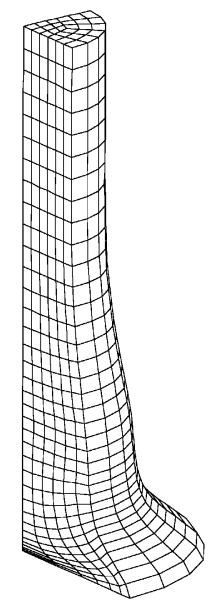

a)

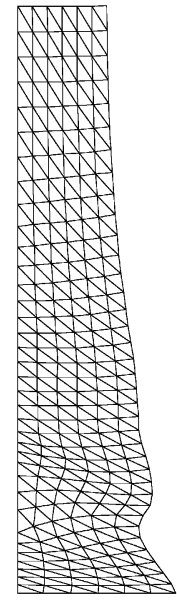

b)

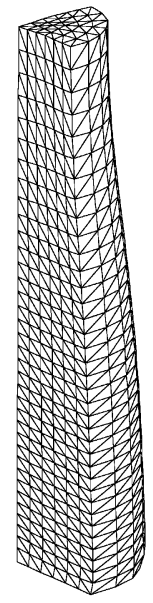

c)

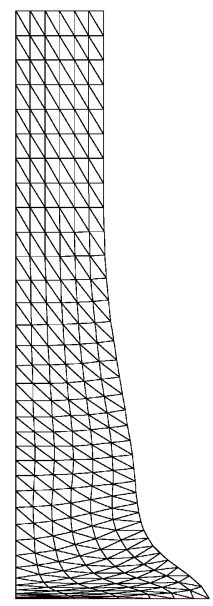

d)

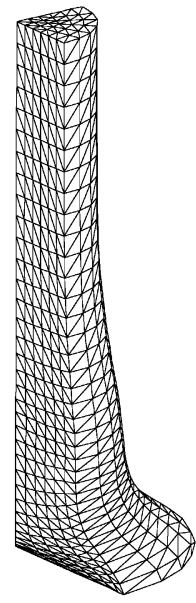

e)

Fig. 2. Deformed shapes of the bar after impact. Different solutions: (a) Q1/P0 hexahedral elements, mixed algorithm; (b) linear triangles, displacement formulation (locking); (c) tetrahedral elements, displacement formulation (locking); (d) linear triangles, split algorithm; (e) tetrahedral elements, split algorithm. 


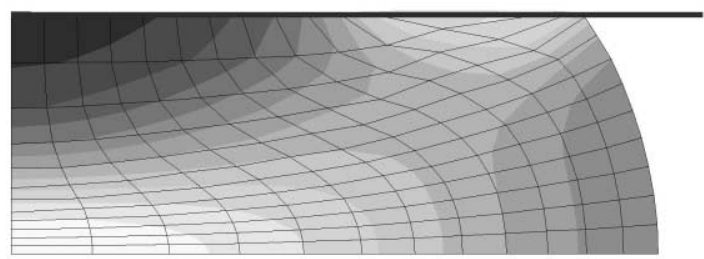

a)

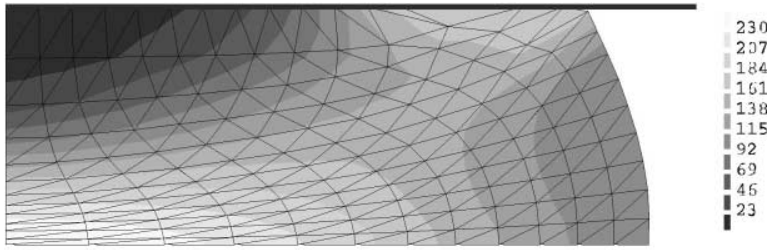

b)

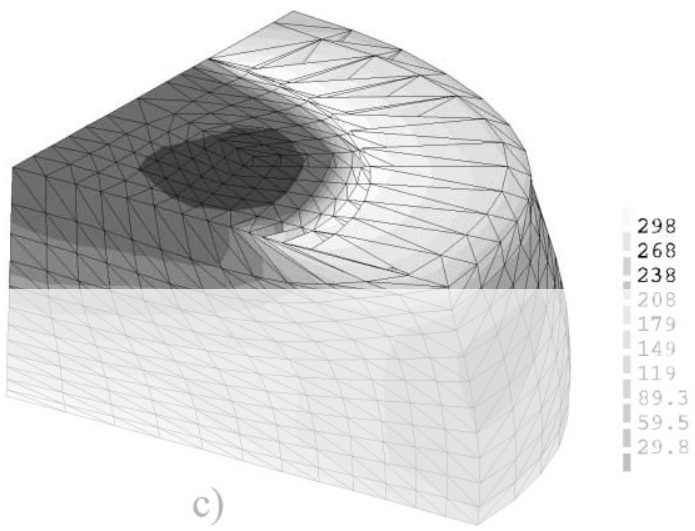

Fig. 3. Upsetting of a cylindrical billet — deformed shape at $60 \%$ upsetting with the temperature distribution: (a) quadrilaterals, mixed formulation; (b) triangles; (c) tetrahedra, split algorithm.
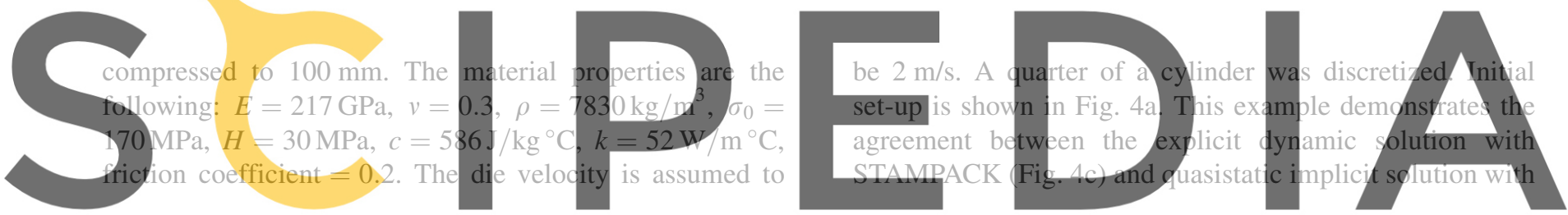

Register for free at https//www.scipedia.com to download the version without the watermark

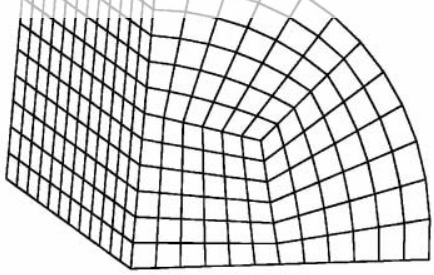

a)

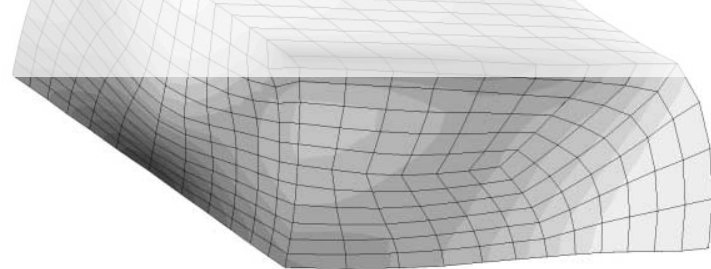

b)
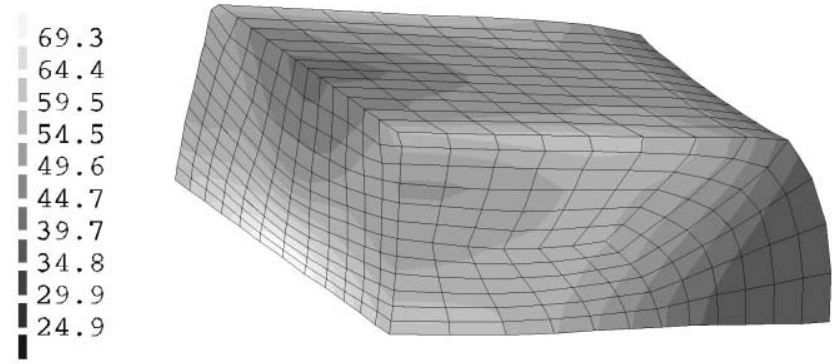

c)

Fig. 4. Sidepressing of a cylinder: (a) initial mesh; (b) temperature distribution — implicit solution (ABAQUS); (c) temperature distribution — explicit solution (STAMPACK). 
ABAQUS (Fig. 4b). Far more efficient is the explicit solution, it took 18 min CPU on Pentium II $350 \mathrm{MHz}$, while the implicit analysis took about $8 \mathrm{~h}$ CPU on Cray J916. Unfortunately, we do not have the comparisons for the same platform, but this demonstrates the computational efficiency of the explicit solution in comparison with the implicit one.

\section{Concluding remarks}

The explicit thermomechanical algorithm and the split algorithm, implemented in the finite element explicit program STAMPACK have given good results for benchmark examples of bulk forming. A good agreement between implicit quasistatic and explicit dynamic results have been observed, at the same time in 3D analysis a considerable advantage of explicit program has been seen. This allows us to see good perspectives in the use of our explicit code in the simulation of industrial problems of bulk forming. For this purpose, further development of the software, including adaptive remeshing, is planned.

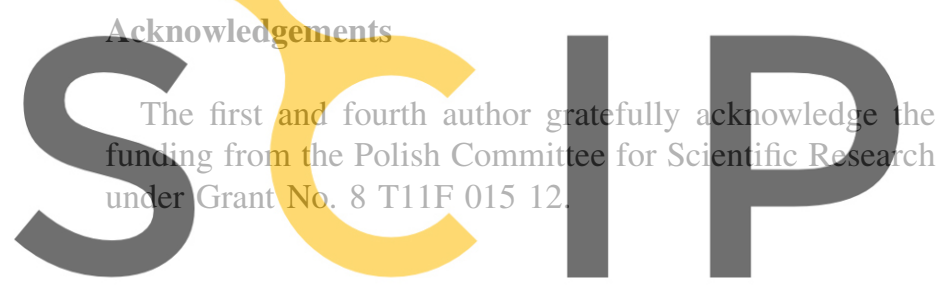

References

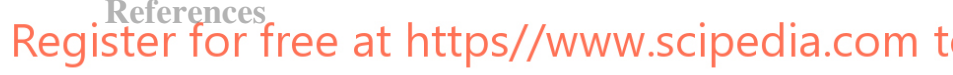

[1] J. Bonet, A.J. Burton, A simple average nodal pressure tetrahedral element for incompressible and nearly incompressible dynamic explicit applications, Commun. Num. Meth. Eng. 14 (1998) 437-449.

[2] C.G. Garino, J. Oliver, Use of a large strain elastoplastic model for simulation of metal forming processes, in: J.L. Chenot, R. Wood, O.C. Zienkiewicz (Eds.), NUMIFORM '92, Balkema, 1992.

[3] C.G. Garino, J. Rojek, E. Oñate, Simulation of sheet metal stamping processes using a solid finite strain model, in: L.A. Godoy, S.R. Idelsohn, P.A.A. Laura, D.T. Mook (Eds.), Applied Mechanics in the Americas, Vol. 1, Santa Fe, Argentina, 1995, pp. 97-102.

[4] T.J.R. Hughes, The Finite Element Method. Linear Static and Dynamic Finite Element Analysis, Prentice-Hall, Englewood Cliffs, NJ 1987.

[5] T.J.R. Hughes, L.P. Franca, M. Balestre, A new finite element formulation for computational fluid dynamics. V. Circumventing the Babuska-Brezzi condition: a stable Petrov-Galerkin formulation for the Stokes problem accommodating equal-order interpolations, Comput. Meth. Appl. Mech. Eng. 59 (1986).

[6] H. Lippmann, Metal Forming Plasticity, Springer, Berlin, 1979

[7] J.C. Simo, M. Ortiz, A unified approach to finite deformation. elastoplastic analysis based on the use of hyperelastic constitutive relations, Comput. Meth. Appl. Mech. Eng. 49 (1985).

[8] P. Wriggers, C. Miehe, M. Kleiber, J.C. Simo, On the coupled thermomechanical treatment of necking problems via finite element methods, Int. J. Num. Meth. Eng. 33 (1992) 869-883.

[9] O.C. Zienkiewicz, R.L. Taylor, The Finite Element Method, 4th Edition, Vol. 1, McGraw-Hill, London, 1989.

[10] O.C. Zienkiewicz, R.L. Taylor, The Finite Element Method, 4th

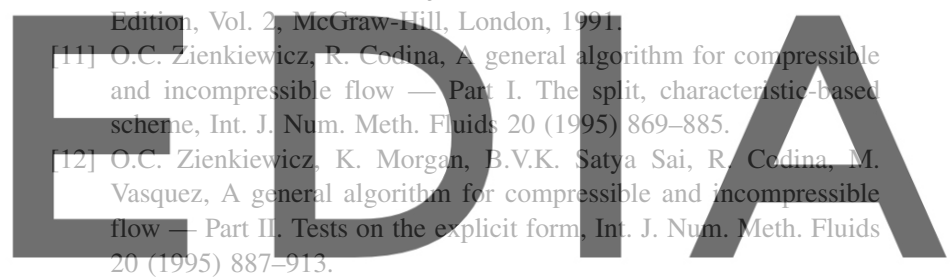

[13] O.C. Zienkiewicz, J. Rojek, R.L. Taylor, M. Pastor, Triangles and

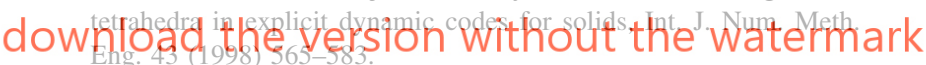

[14] ABAQUS, Example Manual, Version 5.6, Hibbit, Karlsson \& Sorensen, Inc., 1996. 